\title{
Empowering citizens towards the co-creation of sustainable cities
}

\author{
Verónica Gutiérrez, Dimitrios Amaxilatis, Georgios Mylonas, and Luis Muñoz Senior Member, IEEE
}

\begin{abstract}
Urban ecosystems are becoming one of the most potentially attractive scenarios for innovating new services and technologies. In parallel, city managers, urban utilities and other stakeholders are fostering the intensive use of advanced technologies aiming at improving present city performance and sustainability. The deployment of such technology entails the generation of massive amounts of information which in many cases might become useful for other services and applications. Hence, aiming at taking advantage of such massive amounts of information and deployed technology as well as breaking down the potential digital barrier, some easy-to-use tools have to be made available to the urban stakeholders. These tools integrated in a platform, operated directly or indirectly by the city, provide a singular opportunity for exploiting the concept of connected city whilst promoting innovation in all city dimensions and making the co-creation concept a reality, with an eventual impact on government policies.
\end{abstract}

Index Terms-co-creation, IoT, experimentation, ecosystem, OrganiCity, services, smart cities, urban platform

\section{INTRODUCTION}

$\mathrm{I}^{\mathrm{n}}$ nternet of Things (IoT) technology is steadily becoming an omnipresent reality, leveraging connected ecosystems where ubiquitous connectivity and big data applications are enabling innovation all around the world. It is marketed like a panacea, being employed in many application fields including, among others, smart home solutions, self-driving cars as the future of the automotive industry and smart cities.

Nowadays, most people in Europe live and work in cities and soon, it is expected that the population living in urban environments will further increase. Thus, cities will need to deal with the growing demands for services of their citizens and visitors while their urban infrastructures naturally age and become outdated.

Information and Communication Technologies (ICT) will play an increasingly important role in the transformation of cities in the forthcoming decades. Some rationality in the use of technology needs to be applied by decision makers when defining their future strategies. More sustainable management of the city services such as transportation, energy and waste

Veronica Gutiérrez is with the Telefonica, Santander 39003 Spain (e-mail: veronica.gutierrezpolidura@telefonica.com). She carried out her contribution when working at Dept. of Communications Engineering, University of Cantabria, Santander-Spain.

Copyright (c) 2012 IEEE. Personal use of this material is permitted. However, permission to use this material for any other purposes must be obtained from the IEEE by sending a request to pubs-permissions@ieee.org. management, health and social care for dependent people is required, avoiding the creation of an unsustainable sea of vertical solutions for every urban service. For this purpose, the use of the Future Internet (FI) together with IoT technologies is considered an enabler to foster the transformation towards the smart city paradigm.

A prerequisite for building smarter cities is the participation of society. The involvement and education of the different stakeholders in the new initiatives need to be considered from the very beginning to consolidate the different communities within the city innovation ecosystem. To this end, cities need to develop their own smart city vision, defining the strategies to engage and mobilize final users of their services to participate in the new initiatives. In parallel, they should also be part of the ongoing discussions for the development of their local environment.

No urban environment is the same, since each city comes with its own demographic, social, cultural and geographical contexts and challenges. The diversity of potential areas of application for IoT and FI technologies and the complex interdependencies between the urban services within the cities makes it difficult to find a single, truly scalable, pragmatic and applicable solution, which enables the management of the city services together with the stimulation of a co-creation ecosystem. In this article, the Experimentation as a Service (EaaS) framework conceived within the OrganiCity project [1] is presented as an option to tackle this challenge.

The work is organized as follows: section II describes the urban ecosystems, where the IoT infrastructures appear as enablers for the sustainable management of smart cities through the urban platform. In section III, the OrganiCity Experimentation as a Service concept is presented, describing the co-creation process with the different stakeholders and the different components that have been developed to create more inclusive innovation ecosystems. The results of two different experiments are presented in Section IV in order to validate the approach and illustrate how the co-creation tools help users with different skills to carry out their experiments obtaining interesting results, and also how cities with or without existing IoT infrastructure are aided by OrganiCity. Finally, Section V provides the main conclusions, along with several future steps.

Dimitrios Amaxilatis is with the Computer Technology Institute and Press, "Diophantus", Patras 26504 Greece (e-mail: amaxilat@cti.gr).

Georgios Mylonas is with the Computer Technology Institute and Press, "Diophantus", Patras 26504 Greece (e-mail: mylonasg@cti.gr).

Luis Muñoz is with the Communications Engineering Department, University of Cantabria, Santander 39005 Spain (e-mail: luis@tlmat.unican.es). 


\section{THE URBAN ECOSYSTEM}

In this section, we explain how the use of IoT and FI technologies are clear candidates to enable the consolidation of urban ecosystems within cities.

\section{A. IoT infrastructure as an enabler for setting up the smart city paradigm}

While ICT is in the heart of many modern cities, existing infrastructures need to be revamped to enable new ways to monitor and control urban services. This should be accompanied by the installation of new IoT infrastructures, as basic components for the integrated management of the city.

One essential requirement for the deployment of sustainable infrastructures is to handle the traditional inefficient use of communication infrastructures. Due to the broad range of heterogeneous solutions available in cities, a level of abstraction of the data processed is required in order to guarantee the interoperability of the information generated by IoT devices of different vendors and applications, independently and regardless of the underlying technologies.

Setting up an IoT infrastructure often entails the deployment of a huge number of devices to support various urban services. Although this will enable the development of novel solutions with a positive impact on the quality of city services, one of the major challenges for the cities in the future will be how to manage the increasing number of these heterogeneous and geographically dispersed devices. Maintenance of the IoT infrastructure should be included as part of the strategy towards future smarter cities. Additionally, IoT infrastructures within cities can be augmented with citizen participation [2]. Using applications on their smartphones or wearables, citizens will not only be consumers of digital services, but will also generate data as producers within possible smart city scenarios.

Finally, the definition and use of common and harmonized data models, using semantic descriptions, enables the conception of new innovative solutions to interact with city services and to manage the urban environment, which can be replicable in other urban ecosystems.

\section{B. Services and citizen engagement}

Cities can be considered complex systems, where a wide range of urban services are provided to the different stakeholders, organizations and businesses. The design and implementation of smart vision embraces technological, economical and sociological developments within the cities, where citizens play a major role.

Progress towards citizens' better quality of life is a goal shared by every city. Moreover, cities need to improve the operational efficiency of urban services. Hence, a better understanding of cities is required, carrying out an analysis of urban services in order to extract tangible requirements for their sustainable management; both local authorities and other stakeholders need to collaborate and their involvement will be crucial to success in the implementation of holistic management of cities.

Moreover, the intensive use of technology within all the facets of the city should empower smarter citizens. To this end, cities are obliged to encompass the deployment of new IoT infrastructures with the development of specific plans for people to become aware of the progress towards the smart city. They should engage and motivate citizens to participate, as they are the natural influencers of the future co-creation process within the urban landscape. Apart from empowering them to make smarter decisions and personal choices in their daily lives, this could be a way in which citizens increase their ownership sense and pride in belonging to the cities, having a better understanding of their neighborhoods.

\section{The urban platform}

Apart from the deployment of new IoT infrastructures and citizen participation initiatives, sustainable management of smart cities demands a platform that harmonizes their urban infrastructures and services. This is what we call the Urban Platform, made up of vertical pillars linked to the city services.

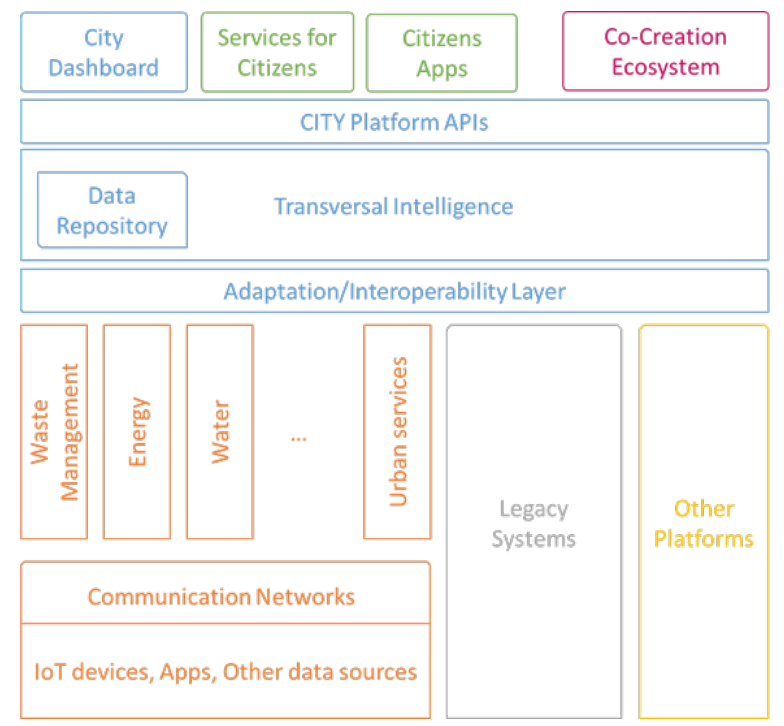

Fig. 1. Urban platform for the sustainable management of smart cities

Fig. 1 presents a high-level architecture of the Urban Platform, addressing the holistic vision of smart city management. As can be seen, legacy systems, existing platforms or other IoT-enabled services need to coexist within the same platform. The classic approach, where services are provided in a vertical manner, is discarded to avoid the creation of isolated silos, because innovation is constrained in that way. The adaptation of urban data streams to a common data model that harmonizes and guarantees the interoperability among the city services is fundamental for building smarter cities. To achieve this, on top of the aforementioned services and solutions, the Urban Platform includes an adaptation/interoperability layer that deals with the integration of data streams and incoming events from lower layers, so they can be stored within a common Data Repository. The Transversal Intelligence layer is placed in the upper tier, allowing cities to implement business logic for provision of real intelligence for smart cities. The implementation of this logic depends on the context of each city, as it needs to consider the traditional management of urban services and the ways predictive and decision models can be implemented to create new rules for integrated city management. On top of that, the 
Urban Platform proposes the creation of an open city environment, with common and well-established APIs, enabling the implementation of city dashboards, the development of useful services and applications for citizens, as well as development of enablers for consolidating the innovation ecosystem.

The development of the smart city vision, together with the implementation of the Urban platform is a process that will take several years. It requires the constant involvement of the different stakeholders and coordinated actions to overcome the different obstacles that might appear in the process.

In Europe, cities often delegate the provision of local services to private companies, aiming to reduce their operational costs and improve the quality of the services offered. Companies have the exclusive right to run the urban services in specific areas of the city, as they have been awarded by competitive tender. However, if the adoption of IoT technologies is not included in the contracts with the urban providers, cities should invest part of their budget to that end. As these types of contracts last for many years, inappropriate decisions can hinder the development towards the smart city paradigm. Every city is handling this challenge, developing different actions. As an example, in Santander, Spain, every time a competitive tender to subcontract urban services is instigated, the city elaborates public procurements in which, it is mandatory to update the existing infrastructure, in order to incorporate and deploy IoT technologies for the management of city services. Moreover, citizen engagement activities are also included in the public procurement, aiming to contribute to the common smart city strategy. These activities allow service providers to create trustable environments, in which both city officials and endusers can interact to improve the existing services.

For supervision of the urban services, it is common that municipalities set up operational and environmental regulations, as well as principles that should be considered as guidelines by the service providers. The Urban Platform can facilitate the work of municipality officials, as within their dashboards, the indicators for the different services can be monitored. Moreover, the use of IoT technologies within the city services and citizen participation through apps can be combined to implement both objective and subjective indicators, which can be measured in order to improve the quality of the services and therefore, citizens' satisfaction.

Although existing regulations within public administrations are slowing down the process in some cities, with all these considerations in mind, we believe that we have identified a solid model for integrated city management that relies on the synergies between the public and private sector. In the next sections, the article focuses on the implementation of the cocreation ecosystem and how the OrganiCity EaaS facility is established in different cities.

\section{EXPERIMENTATION AS A SERVICE: MOBILIZING THE} ECOSYSTEM

\section{A. Rationale}

Traditionally, cities have been the meeting point between societal challenges and technological innovation. The main ambition of OrganiCity(OC) is to make the creation and design of technologies and services for cities more inclusive for any user of the smart city. It tackles the aspects of how smart cities can grow organically with the involvement of different stakeholders (citizens, communities, policy makers, activists, scientists, researchers, developers, city service and technology providers), and not be driven solely by engineering visions.

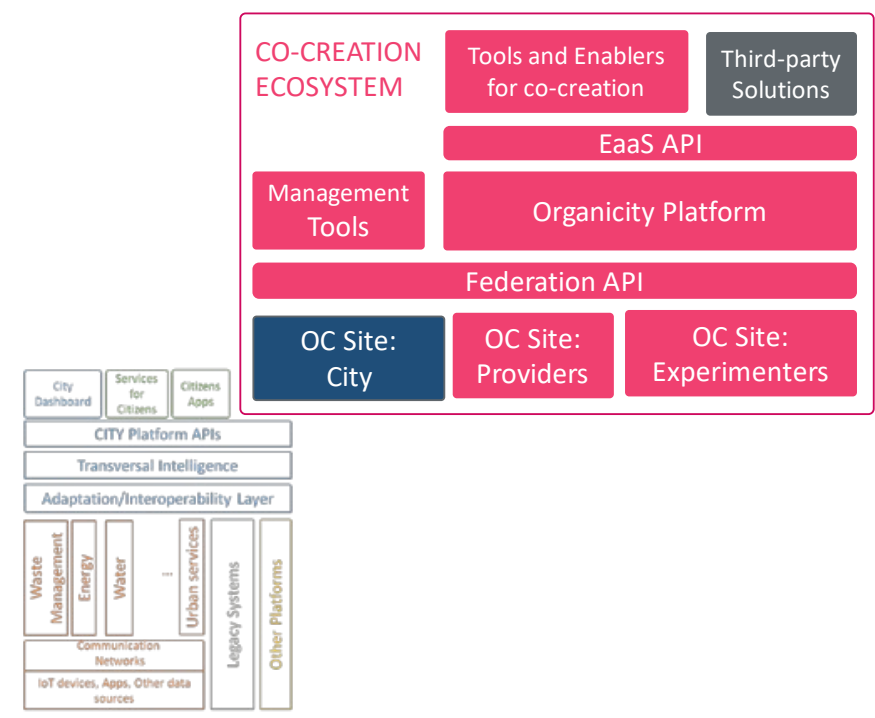

Fig. 2. The co-creation ecosystem of OrganiCity complementing the urban platform for sustainable management of cities

OrganiCity has developed an EaaS facility, as a research and innovation environment for the co-creation of FI-enabled urban knowledge and services. The facility leverages emerging tools and technologies to provide mechanisms that allow users to extract knowledge from different cities, based on the data streams that are generated in the diverse urban ecosystems. To make this more inclusive, the facility delivers a set of tools and enablers to empower any user to be part of the co-creation process. Moreover, the facility provides various means for the engagement of the citizens participating in their validation.

Taking into consideration the resource constraints of cities in times of austerity, it is difficult to create new city infrastructures and/or develop new “experiments”. Setting up the OrganiCity facility on top of the Urban Platform should permit city makers and service providers to address not only the exploration of new pathways for their services, but also to understand any societal implications in a rapid and more iterative manner.

In general, as can be seen in Fig. 2, the Federation API enables cities to integrate different data streams, or any other city asset, into the OrganiCity facility. To this end, they should implement what we call the OC City Site, a component that makes use of the city platform's APIs to gather data from the Urban Platform and feed them as new data assets into the OC facility. To this end, the facility defines a common data model, based on the FIWARE NGSI specification [5] that harmonizes and abstracts the information coming from the federated cities, aiming to guarantee the interoperability of smart city solutions. 


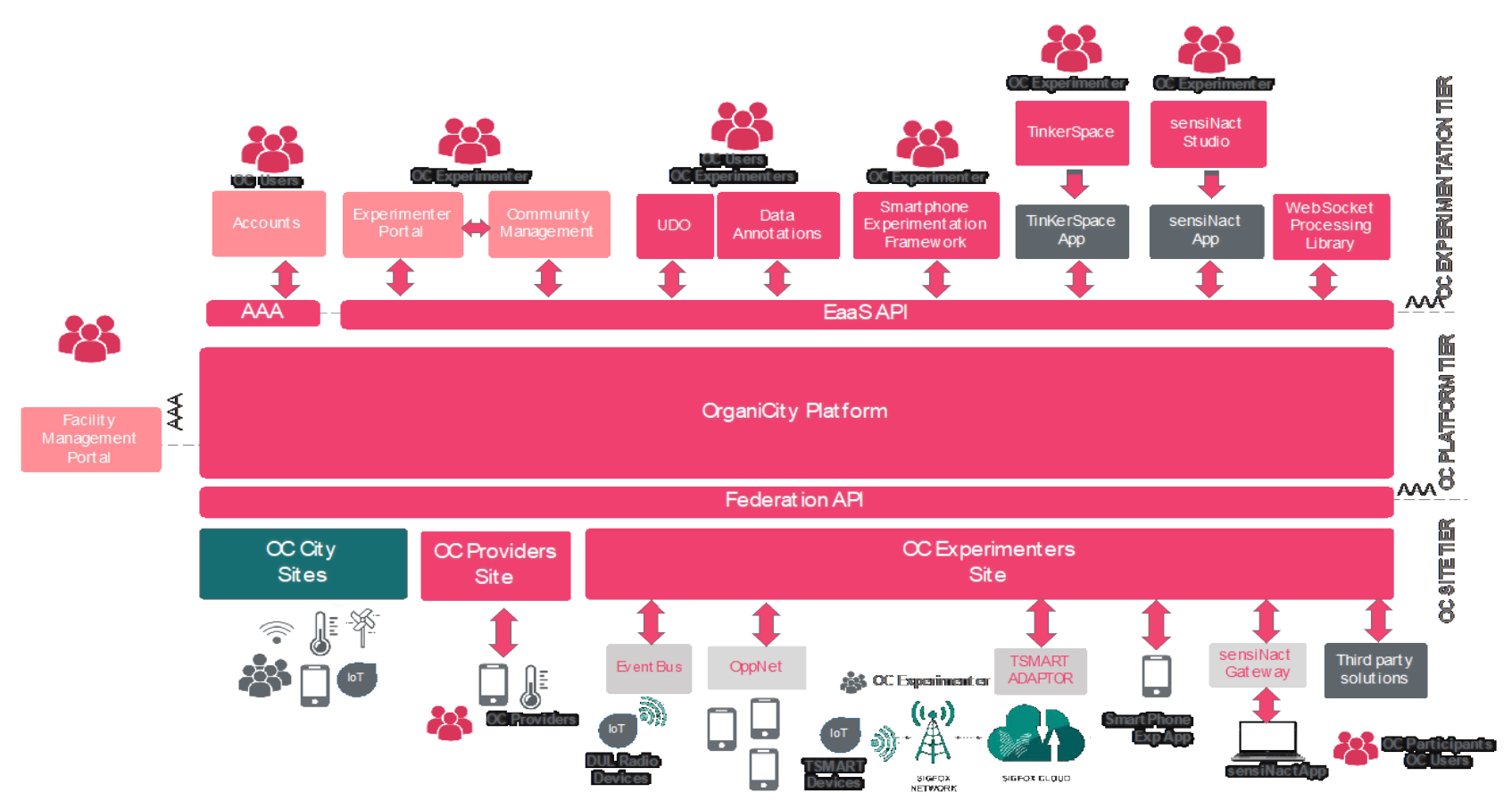

Fig. 3. High-level architecture of the OrganiCity facility

\section{B. ORGANICITY: An EaaS platform}

\section{1) Scope}

OrganiCity offers a flexible EaaS framework, allowing researchers and developers of urban infrastructures, services and knowledge, full exploitation of emerging Future Internet technologies. Research and experimentation on top of the OC facility should lead to the creation of sustainable, effective, and replicable smart city solutions and urban technology developments that are successfully adopted by citizens, local communities and society as a whole.

The design phase of the facility considered questions like how to provide data generated within the cities in a more public domain, whether existing infrastructures are reliable enough for data scale collection with citizens' devices, how creation of actionable knowledge from urban sensors can be scaled, how we can incentivize and technically facilitate effective citizencity-academia-industry co-creation. Different stakeholders such as developers, data analysts, IoT solution manufacturers, urban service providers, activists, sociologists, economists, and citizens were involved, in order to extract requirements for the EaaS facility. As a result, OrganiCity has implemented a set of tools that empowers citizens to be part of the co-creation process at different stages of the urban service lifecycle and provide different means for their participatory engagement.

\section{2) Stakeholders}

The OC facility supports different types of users, permitting them to manage the whole experiment life cycle and offering a set of tools and enablers that make the co-creation and validation of new solutions more inclusive. It can be used remotely by any stakeholder within smart cities.

Depending on the activity that they intend to do, users can request different permissions, supporting the following roles: experimenters, participants, providers, site managers and facility administrators. Experimenters can implement their own solutions using a set of co-creation tools and conduct the corresponding experiment. To validate the solutions, they can invite other participants within the scope of the experiment (e.g., using an application developed by the experimenter). Providers are users who do not belong to any experiment, but want to contribute with crowdsourced data to the facility (e.g., feed data from their own weather stations). Facility administrators deal with the management of the entire platform, being able to federate new urban ecosystems within the cities, assigning permissions and roles to other users, configuring the facility parameters and monitoring its activity. Finally, site managers are users belonging to the federated cities that can configure the information related to the urban services, the tags that can be used for annotating the data assets, and so on.

The use of the facility and its tools within the urban ecosystems will guarantee that communities of users can grow around emerging experimental technologies. Experimenters and service providers can leverage active contributors and early technology adopters. These communities will not only inform and contribute to the design of technology, but also provide a basis for exploring new business models derived from the emerging solutions.

\section{3) Architecture}

The OrganiCity platform uses the Microservices Architecture pattern [6]. Each service deployed in the context of OrganiCity is a standalone application that uses a central authentication service [7] (based on OAuth2.0) and provides either a user interface for end-users or a programmatic API (usually RESTful). This allows us to develop different applications based on their requirements and using tools, programming 
languages and frameworks that best suit each case (i.e., node.js for frontend applications, Java for backend services). Additionally, the microservices pattern allows us to better fine tune different parts of our infrastructure based on usage at runtime levels and thus provide better user experience and responsiveness to any number of clients, a major requirement for big cross-city IoT applications.

\section{4) Components}

Instead of building the EaaS facility from scratch, the project has reused components and experiences from three well established European urban ecosystems: Aarhus (Denmark), London (UK) and Santander (Spain), which are developing different smart city initiatives and already have infrastructures in place. Fig. 3 depicts the high-level architecture of the OC facility, in which 3 different tiers can be identified: the OC Site Tier, the OC Platform Tier and the OC experimentation Tier.

On top of the OrganiCity architecture, the OC Experimentation Tier offers a set of tools and enablers to be used within the experiments. Practical guidance about the use of the facility is available in [7]. First, through the Accounts component, citizens or any other stakeholder can register as OrganiCity users. OC users can request the "providers" or "experimenters" role, depending on whether they want just to provide data to the facility, or to carry out an experiment.

For novel experimenters and other stakeholders, OC has developed the concept of the Urban Data Observatory (UDO), a component that allows users to explore and visualize mixed static and real-time urban and social data streams, enabling the exploitation of such data. To this end, the UDO relies on the EaaS APIs [8] provided by the OC Platform. More precisely, it uses the Asset Discovery Service and the Data Annotation Service, which provide support for crowdsourcing annotations of the urban data assets using the well-established social media paradigm of tags. In more detail, through the UDO, users can add the appropriate annotation tags to the assets they browse or view the tags other users have already assigned. In the background, the UDO is communicating via a well-defined API with the Data Annotation Service to retrieve, add or update existing tags assigned to each asset. Based on that data. On a second level, experimenters can identify real world events or generate further insights within the smart city by observing the tags assigned to each asset.

Additionally, OrganiCity provides a set of co-creation tools [9] such as SensiNact Studio, TinkerSpace, and Smartphone Experimentation that facilitate the exploration of data within the urban ecosystems, as well as the development of new services and applications using existing data sources. The tools support users from IoT developers to non-tech-savvy activists, giving them the opportunity to define their application logic as actions to be taken when specific events occur, or even create their own applications from scratch. At the same time, it helps them understand why open data, smart cities, IoT, crowd sourcing, big data, and other related topics are important for the sustainability of cities.

The core component of the facility is the OrganiCity platform that can be found in the OC Platform Tier. Based on the use of the Orion Context Broker Generic Enabler [10], it implements what we call the Asset Directory, a catalogue that contains all available data sources integrated in the platform. This component integrates urban data streams of the federated cities, as well as crowdsourced data generated by both providers and experimenters, as data assets in OrganiCity. Besides the basic discovery functionalities, it also acts as a data exchange hub, which allows other components of the facility to subscribe to updates of the available data and provides pointers to access historical data when and from where they are available. Moreover, the OC platform has been designed to be easily configured through a dedicated Facility Management Portal that acts as a central control point for administrators of OrganiCity and service managers of the federated cities.

Finally, within the OC Site Tier, different components enable the generation of data. Each OC City Site integrates its urban data streams generated within the IoT infrastructures, data available on Open Data platforms (e.g., Comprehensive Knowledge Archive Network, CKAN) or any other existing service through their respective APIs (e.g., API provided by the Public Bus Company in Santander). To proceed with integration, every city willing to be federated within OrganiCity needs to deploy its own OC City Site adaptors, so that the Federation API of OrganiCity is able to consume the data in the central OrganiCity infrastructure. In the case of experimenters/participants or providers, the OC experimenters Site and the OC providers Site respectively enable the injection of data into the platform. To ease this process, OrganiCity utilizes a set of hardware platforms such as TSmart, and DULradio that are already integrated, reducing the need for integration or development of custom software. Additionally, the Smartphone Experimentation Tool is designed to allow participants to take part in data collection experiments inside the cities, feeding data collected by the sensors integrated in smartphones, or other IoT devices attached to them (e.g., wearables over Bluetooth LE).

\section{VALIDATION}

To illustrate how the different stakeholders can benefit from the OrganiCity facility, two different experiments have been carried out. The first one has been conducted in collaboration with the officials of the Climate Change Office in Santander (Spain). They have studied the Urban Heat Island (UHI) effect in the city [11] during winter and summer based on the data collected by IoT devices installed in public vehicles, which is available in OrganiCity. The second one is based on the use of the Smartphone Experimentation co-creation tool. In this experiment, the participants have collected data with gas, humidity and temperature sensors attached to their smartphones. Through the Smartphone Experimentation App installed in their devices, the data retrieved in different parts of the city was fed to the OC Platform, aiming at conducting a similar study in the city of Patras (Greece), in a scenario where no related IoT city infrastructure is deployed. With these two experiments, we intend to showcase how flexible the OrganiCity framework is when developing and validating distinct solutions under real conditions within smart cities. 


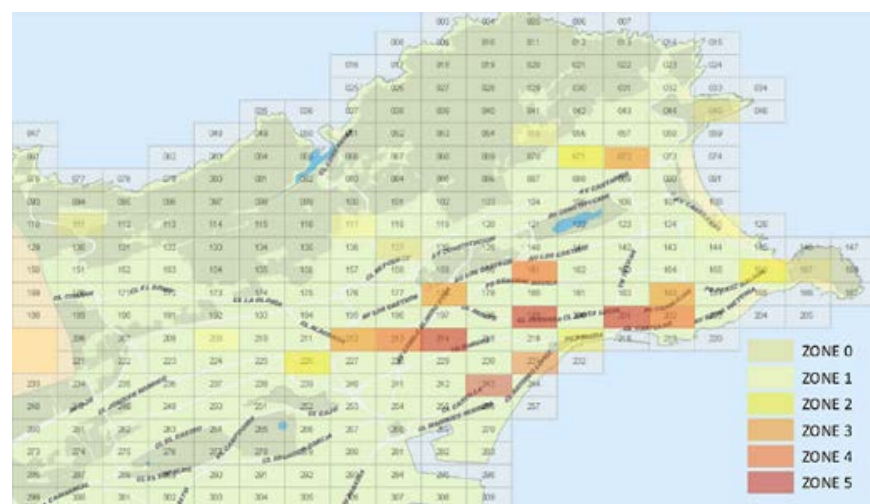

Fig. 4. Experiment information in the Experimenter Portal

\section{A. Analysis of the Urban Heat Island Effect}

Many urban areas experience elevated temperatures compared with their rural surroundings; this difference in temperature being the basis for the formation of a UHI. Due to human activity, the mean air temperature in the city is several degrees warmer than the surroundings, this effect being less intense in smaller cities.

To identify heat islands within the cities, different methods and models can be applied. In this work, we rely on data collected by environmental sensors installed in nearly 150 urban transportation buses and in park and garden vehicles moving around the city of Santander. The information collected by these sensors when moving in the different city routes is transmitted (relying on General Packet Radio Service, GPRS, infrastructure) and fed to the OrganiCity facility by means of the corresponding EaaS API.

The experiment consists in assessing the thermic load qualitative model adopted by the Santander Local Climate Change Office, which categorizes the city in different climate zones according to the probability of the urban heat island effect $[12,13]$ in these areas.

The methodology applied is as follows. Firstly, within the city area, a grid with cells of 500x250 meters was created, as depicted in Fig. 4. Taking into account several factors related to the vegetative cover and surface properties in each one of the cells, and according to the model adopted, the cells were categorized into 6 climate zones: rural (zone 0 - null warm-up), parks (zone 1 - residual warm-up), sub-urban residential (zone 2 - low warm-up), commercial (zone 3 -intermediate warm-up), urban residential (zone 4 - intermediate/high warm-up) and

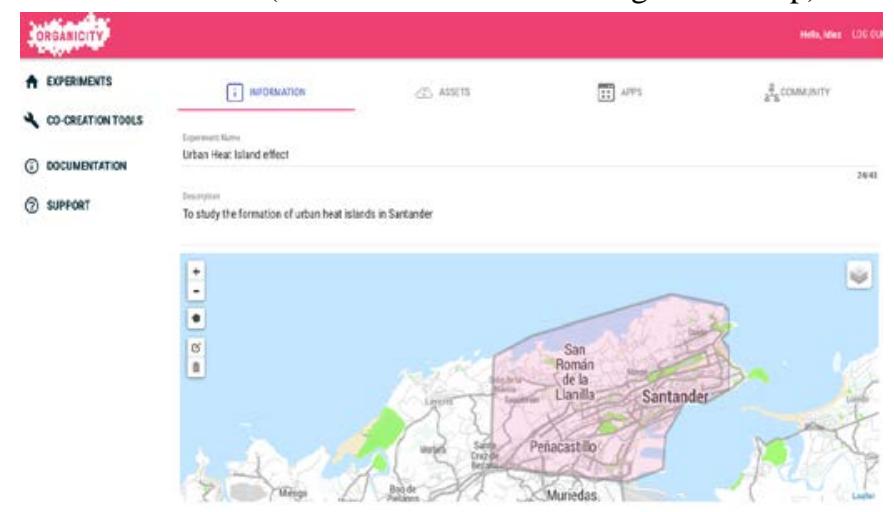

Fig. 5. Santander zones used for the experiment analysis downtown (zone 5 - high warm-up). Furthermore, multiple cells fitting the most commonly adopted city profile (multiple areas categorized as Zone 0-Zone 2-Zone 3-Zone 5-Zone 4-Zone 1Zone 2-Zone 0 according to the climate zone model) are chosen [11]. Secondly, to carry out the study, we created a new experiment in the Experimenter Portal, defining its scope within the Santander area (see Fig. 5). Then, we selected the data assets

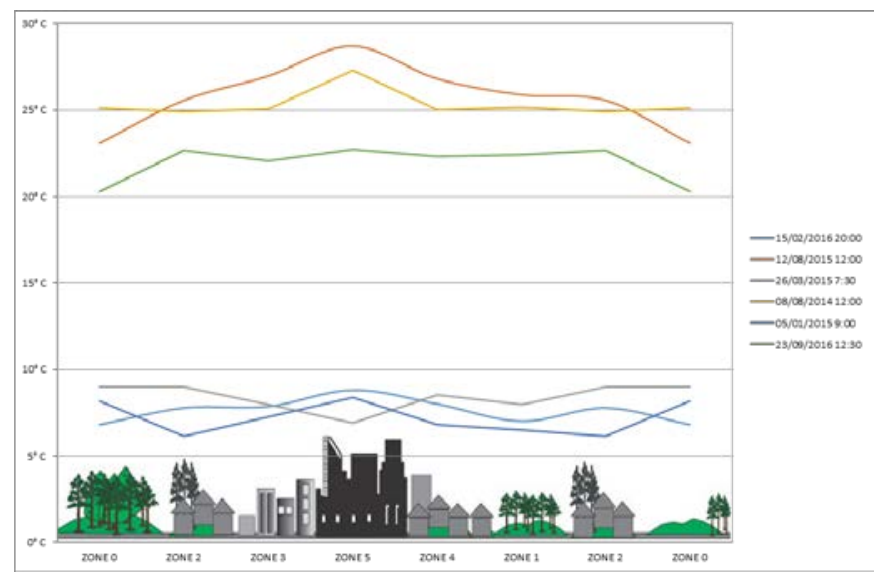

Fig. 6. UHI effect comparison in Santander (in ${ }^{\circ} \mathrm{C}$ )

used within the experiment; that is, those corresponding to the ones collected by the sensors deployed in public buses and vehicles, which provide temperature values as part of the environmental monitoring service in Santander. By means of the EaaS APIs, historical data for different periods was retrieved. Further data processing was done to obtain representative samples of temperature measurements in at least 4 cells for each one of the aforementioned climate zones.

Aiming at assessing the matching between the thermic load qualitative model and the collected measurements, the temperature variation per climate zone during one of the warmest days in the summer period and during one of the coldest days in the winter period was obtained during the years 2014, 2015 and 2016. This is shown in Fig. 6.

As can be seen, the highest temperature difference between zone 5 (downtown) and zone 0 (rural zones located in the surroundings of Santander) is around $6^{\circ} \mathrm{C}$ during the summer period, while in winter time this difference is $2^{\circ} \mathrm{C}$. Hence, the results show the good matching between the thermic load qualitative model and the collected measurements, which should facilitate the prediction of the urban heat island effect in different city zones.

\section{B. Citizen participation through smart phones}

The experiment carried out in Patras (Greece) showcases the smartphone crowdsourcing capabilities of OrganiCity, while demonstrating how an experiment similar to the one carried out in Santander, a city with extensive IoT infrastructure, can be carried out in a location with no IoT infrastructure. The experiment monitors nitrogen dioxide $\left(\mathrm{NO}_{2}\right)$ concentration levels in various parts of the urban landscape of the city. This gas is specifically related to diesel engine emissions, wood use in fireplaces, and other similar human activity.

To that end, we defined a new OrganiCity experiment to be carried out in Patras through the Experimentation Portal. It offers additional capabilities provided for monitoring the progress of the experiment, since these experiments are based 
on Android software components provided by the project itself, in the form of the Smartphone Experimentation Portal (see Fig. 7). Citizens that wish to contribute to the project can install the OrganiCity Android app, which essentially provides both a sensing instrument, through the device's integrated sensors, and an IoT "proxy", by forwarding measurements from connected devices. In this case, we use the second scenario, since there is no smartphone device available with an integrated $\mathrm{NO}_{2}$ sensor. The OrganiCity Android app [14] downloads the "experiment" to be executed, and, when the owner gives permission to the app to use e.g., WiFi and Bluetooth, it transparently handles all aspects related to collecting measurements and forwarding them to OrganiCity.

In order to provide a sensing endpoint for the experiment, we constructed an IoT "box" (see Fig. 8), to be carried by citizens during their daily commute. It has an integrated battery and communicates with an Android smartphone running the OrganiCity app over Bluetooth LE. The IoT box used is based on the following hardware parts:

- Blend Micro [15], a popular microcontroller.

- Grove Multichannel Gas Sensor and Grove

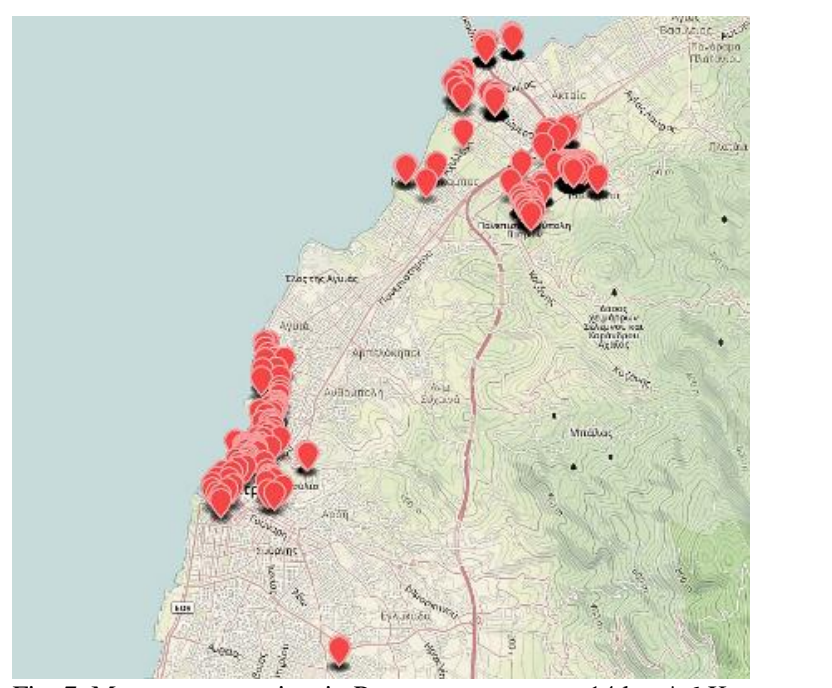

Fig. 7. Measurement points in Patras - area approx. $14 \mathrm{~km} * 6 \mathrm{Km}$

Temperature and Humidity IR Sensor [16].

- $\quad$ Li-Po Rider and LiPo Battery - 2200mAh 3.7V.

- A custom PCB board integrating the above.

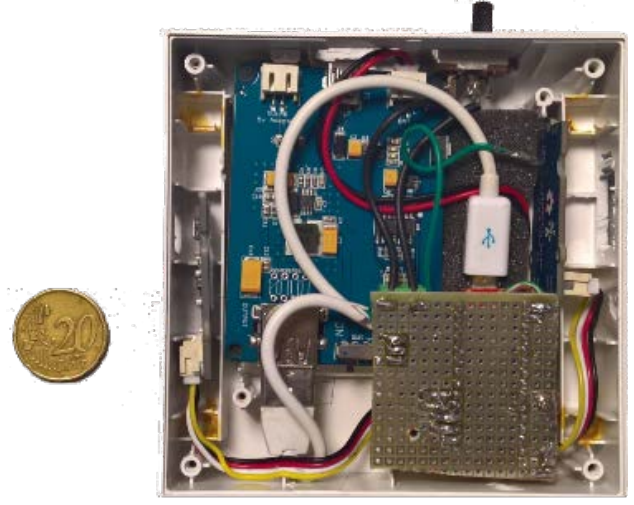

Fig. 8. The IoT box hardware used in Patras' experiment
Delving into additional details regarding the implementation of the code executed on the smartphone and the sensor box, the Android part of the code can be found online in the respective GitHub repository ${ }^{1}$. In essence, developers utilize a provided code skeleton, extending Java classes and implementing a few methods for handling the communication with the sensor box over BLE, and the bundling of measurements as OrganiCity readings. The handling of the readings, i.e., formatting, checking and uploading to OrganiCity's servers is done automatically by the Android app, thus the developers do not need to write code for these tasks. The additional lines of code required in this case are in the order of 40-50 lines in total.

\author{
Sensor Box pseudocode \\ Set Up BLE communication and Gas Sensors \\ Power On Gas Sensor \\ while(TRUE) \{ \\ if ("BLE Terminal is connected") \{ \\ while ("BLE Terminal Connected") \{ \\ send "START" \\ if message received send "FINISH" \\ \} \\ else disconnect \\ \} \\ else Read sensors every 30 seconds \\ \} \\ Read the sensor values: \\ \{ \\ Read Gas Sensor and power off \\ Power On Temp-humid Sensor, read value, power off \\ \} \\ Power On Multichannel Gas Sensor
}

\section{BLE smartphone plugin pseudocode}

Every 2 minutes:

Close previous connection if open and reset

Scan for and create a list of discovered BLE devices

while (Array List of BLE devices != empty)

\{

Connect with first BLE device on the list

while (BLE Device connected)

\{

Waiting for BLE device message

if (message $==$ START)

send message $=$ Send a New Sensor Value

else if (message $=$ New Sensor Value)

update Sensor Values

\}

\}

delete BLE device from list

Regarding the experiment plugin, developers have to implement a "handleContextRequest" method, used by the framework each time an updated sensor value is needed. This is

\footnotetext{
${ }^{1}$ Organicity GitHub repository, experiment plugins, https://github.com/OrganicityEu/smartphone-experimentation-experiments
} 
usually done every 30 - 60 seconds, but times may vary based on the experiment.

\author{
handleContextRequest pseudocode \\ create an Array List for readings \\ if (experiment exists) \\ create new reading from values of BLE plugin \\ add reading to the app internal database \\ wait for new event
}

Over the course of 7 days in winter and 5 days in summer, we produced over 1400 measurements with the box, covering a large part of the city center. In this case, the usual method of producing gas concentration readings is to monitor the air at specific points for a period of 30 minutes, 1 hour or 24 hours and then calculate average values. We opted to measure with the IoT box while people were on the move, simulating real-life conditions.

Moreover, our sensors were calibrated with a rough estimation, and do not provide the same accuracy as more expensive ones. We utilized air pollution monitoring station readings provided by the local government to make our calibration. However, our goal was to highlight that even with the use of simple equipment, we can reveal certain trends and that OrganiCity can help e.g., interested citizen groups and provide a good foundation for such initiatives. Our results also generally correlate with other studies in the area [17].

Overall, the experiment area covered is partitioned in 9 zones:

- Zone 1, a park with heavy vehicle (trucks) traffic.

- Zone 2, a university campus, suburban area.

- Zone 3, a city area with medium levels of traffic.

- Zone 4, the city center, with heavy traffic and activity.

- Zone 5, a suburban area with heavy traffic.

- Zone 6, a suburban area with medium traffic and lots of houses using fireplaces for heating.

- Zone 7, a central area with very light vehicle traffic.

- Zone 8, a suburban area with heavy traffic and a busy train/bus stop.

- Z Zone 9, a city center area with a beachfront.

The measurements reveal some interesting trends; suburban areas that are more open to wind currents or without traffic produce similar readings. Vehicle traffic plays a major role in nitrogen dioxide concentration; however, it is not the sole producer. Zone 8 is greatly affected by the train and bus traffic, even though it is suburban with no tall buildings in the area. Zone 7 produced the highest readings in winter, probably due to the fact that in Greece many people have reverted to using traditional wood fireplaces for heating in the winter, combined with the high humidity in the area (frequently over 60\%). Zone 1 produces similar readings to city center areas, although it is essentially a park area, due to heavy traffic at certain times. In summertime, we see much lower $\mathrm{NO}_{2}$ levels overall, which seem to correlate with the perceived air pollution levels as well.

Overall, we believe that these IoT experiments can potentially offer great insights e.g., into the current environmental status inside urban areas. OrganiCity can be used

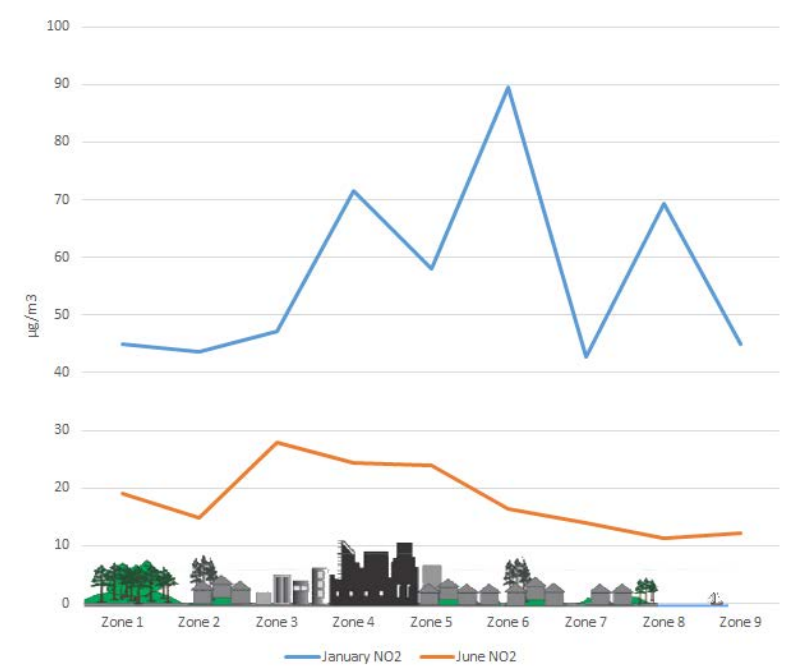

Fig. 9. Nitrogen dioxide concentration measurements in Patras in $\mu \mathrm{g} / \mathrm{m}^{3}$

as the substrate to design, organize and implement such initiatives, even in the case of having no existing IoT infrastructure available.

\section{CONCLUSIONS AND FUTURE WORK}

No two urban environments are the same, as they come with various demographic, social, cultural and geographical contexts, resulting in a great diversity in smart city challenges. Dealing with such challenges will require holistic management of the city by means of what we call urban platforms. The progressive adoption of IoT technologies in urban services will enable the creation of the building blocks for setting up the smart city paradigm. The mobilization and consolidation of innovation ecosystems will be fundamental as it permits us to take charge of tackling diverse city challenges, applying multidisciplinary strategies that can operate along with the traditional management of the city.

The instantiation of the OrganiCity facility, which has been designed to guarantee interoperability with the different Urban platforms, enables research and experimentation with smart city solutions and urban technology developments that have been successfully adopted by citizens, local communities and society as a whole, making the co-creation process more inclusive. Indeed, we believe that in the present digital transformation phase, cities will have to make different facilities available to the citizens, which will provide support to the EaaS paradigm as another urban service and with the same relevance as, for example, public transportation or waste management. Such an approach may become one of the enablers in the consolidation of open government policies or in the reconfiguration of urban ecosystems from the business interaction perspective.

Further to the experiments reported in previous sections, in the near future, we plan to extend these measurements and usecase scenarios to other parts in cities by utilizing the smartphone experimentation framework and experimentation management capabilities provided by OrganiCity, combined with incentive and reward techniques.

\section{ACKNOWLEDGMENT}

This work would not have been possible without the 
contribution of the OrganiCity team, which has been partially funded by the European Union, under the grant agreement No. 645198 of the Horizon 2020 research and innovation program.

\section{REFERENCES}

[1] Organicity project, http://www.organicity.eu

[2] Evangelos Theodoridis, Georgios Mylonas, Verónica Gutiérrez, Luis Muñoz: Large-scale participatory sensing experimentation using smartphones within a smart city. MobiQuitous 2014: 178-187

[3] Verónica Gutiérrez, Evangelos Theodoridis, Georgios Mylonas, Fengrui Shi, Usman Adeel, Luis Diez, Dimitrios Amaxilatis, Johnny Choque, Guillem Camprodon, Julie A. McCann, Luis Muñoz: Co-Creating the Cities of the Future. Sensors 16(11): 1971 (2016)

[4] Dimitrios Amaxilatis, Evangelos Lagoudianakis, Georgios Mylonas, Evangelos Theodoridis: Managing smartphone crowdsensing campaigns through the Organicity smart city platform. UbiComp Adjunct 2016: 1460-1465

[5] FIWARE NGSI specification, http://telefonicaid.github.io/fiwareorion/api/v2/stable/

[6] Microservices pattern, https://en.wikipedia.org/wiki/Microservices

[7] OrganiCity GitHub repository, http://organicityeu.github.io/

[8] OrganiCity Experimentation as a Service APIs, http://api.organicity.eu/

[9] Organicity co-creation tools, http://organicity.eu/tools

[10] Orion Context Broker FIWARE Generic Enabler, https://fiwareorion.readthedocs.io/

[11] U.S. Environmental Protection Agency. 2008. Reducing urban heat islands: Compendium of strategies. Draft. https://www.epa.gov/heatislands/heat-island-compendium

[12] Oke T.R., Boundary Layer Climates, Taylor \& Francis e-library, 2002.

[13] Stewart, I. and Oke, T. R.: Newly developed thermal climate zones for defining and measuring urban heat island magnitude in the canopy layer. Eighth Symposium on the Urban Environment organized by the American Meteorological Society, Phoenix (USA), January 2009, J8.2A.

[14] Organicity Smartphone Experimentation on Google Play store, https://play.google.com/store/apps/details?id=org.ambientdynamix.core

[15] Blend Micro microcontroller, http://redbearlab.com/blendmicro

[16] Grove sensors, http://www.seeedstudio.com/depot/Grove-MultichannelGas-Sensor-p-2502.html

[17] Report on University of Patras campus air quality, pg. 25, http://green.upatras.gr/sites/green.upatras.gr/files/ReviewAtmosphere20 15.pdf

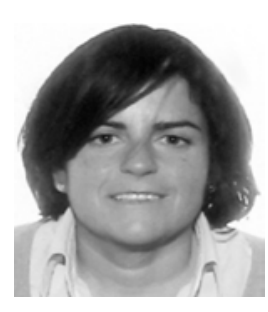

Verónica Gutiérrez was born in Santander, Spain, in 1976. She received the Telecommunications Engineering degree from the University of Cantabria, Spain, in 2000, where has developed most of her professional career, working as senior researcher in the Network Planning and Mobile Communications Laboratory. She has been very active in different European projects framed under the smart city paradigm, such as SmartSantander where she coordinated the development of the citizens' services, the RADICAL project leveraging the Living Lab concept as well as OrganiCity, where she was focused on the design and implementation of the Experimentation as a Service framework. Since March 2017, she moved to Telefonica, where she is leading the project that deals with the implantation of the Smart City platform and the transformation of the urban services in the city of Santander, Spain.

Her research interests are in the development of IoT-based services and applications for smart cities, middleware, experimentation platforms and citizen participation.

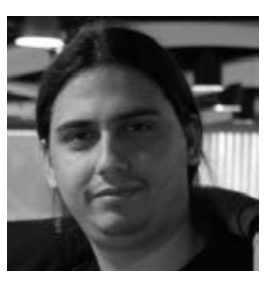

Dimitrios Amaxilatis was born in Kavala, Greece, in 1988. He received the B.S. in Computer Engineering and M.S. in Computer Science from the University of Patras, in 2011 and 2013 and is currently a Ph.D. student in the same University. From 2010 till today, he has been a researcher with the Computer Technology Institute in Patras, Greece. He was also a member of the founding teams of two technological startups in the fields of microprocessor programming (codebender.cc) and home automation (Sensorflare) as well as the Patras City hackerspace (P-Space), the first hackerspace in Greece. His research interests include distributed algorithms, wireless sensor networks, home and building automation, smart city and participatory sensing applications.



Georgios Mylonas is a senior researcher at Computer Technology Institute and Press “Diophantus”. He received his Ph.D., MSc and diploma degree from the Dpt. of Comp. Engineering and Informatics at the University of Patras, Greece. His research interests lie in the areas of wireless sensor networks and distributed systems, IoT and pervasive games. He has been involved in the AEOLUS, WISEBED, Smartsantander, AUDIS, ABC4TRUST and OrganiCity projects, focusing on algorithmic and software issues of wireless sensor networks. He is currently the coordinator of the Green Awareness in Action (GAIA) H2020 project.

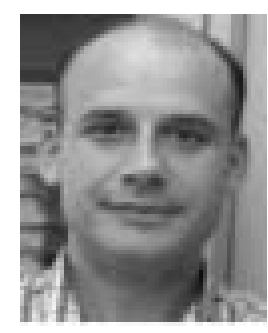

Luis Muñoz (M'95-SM'15) was born in 1964 in Barcelona, Spain. He received both the Telecommunications Engineering degree and Ph.D. from the Polytechnical University of Cataluña (UPC), Spain, in 1990 and 1995, respectively. He also holds a Master in Mathematics, Statistics and Operation Research (Licenciado en Ciencias Matemáticas, UNED). He is head of Network Planning and Mobile Communications at the University of Cantabria, Spain. His research focuses on advanced data transmission techniques, heterogeneous wireless multihop networks, Internet of Things, smart cities and applied mathematical methods for telecommunications. He has participated in several National and European research projects within the 4th, 5th, 6th and 7th Framework Program in which he was the technical manager of SmartSantander. He has published over 150 journal and conference papers. He serves as editor of several journals and he has been invited to participate in the Steering Committee and Technical Program Committee of the most relevant international conferences. In parallel to this activity, he serves as consultant to the Spanish Government as well as for different companies in Europe and the USA. Last but not least, he has served as an expert of the ETSI and European Commission. 